\title{
NEW POLYENIC ANTIBIOTICS ACTIVE AGAINST GRAM-POSITIVE AND -NEGATIVE BACTERIA \\ I. ISOLATION AND PURIFICATION OF ANTIBIOTICS PRODUCED BY GLUCONOBACTER SP. W-315
}

\author{
Toshiniko Watanabe, Kazuo Izaki and Hajime Takahashi \\ The Department of Agricultural Chemistry, Faculty of Agriculture, \\ Tohoku University, Sendai 980, Japan \\ (Received for publication March 12, 1982)
}

\begin{abstract}
A new antibiotic, tentatively named as AB-315, was isolated from the fermentation broth of Gluconobacter sp. W-315. The antibiotic consists of a mixture of chemically related compounds. These compounds showed similar profiles in UV absorbancy. The antibiotics were active against Gram-positive and -negative bacteria, slightly active against fungi but not against yeasts.
\end{abstract}

During the course of search for new antifungal antibiotics, a bacterial strain W-315 isolated from a soil sample collected in this faculty was found to produce antibiotics which are active against bacteria and slightly active against fungi. The strain W-315 was found to belong to genus Gluconobacter and was named as Gluconobacter sp. W-315 ${ }^{11}$. The antibiotic, designated AB-315, gave yellow needle crystals and was shown to be a mixture of carbonyl-conjugated pentaene (manuscript in preparation). As the result of comparative studies on the antibiotic with the other known antibiotics, it was shown to be a mixture of new polyenic antibiotics active against Gram-positive and -negative bacteria, and slightly active against fungi but not against yeasts.

In this paper, we describe the isolation, purification and properties of the antibiotics.

\section{Materials and Methods}

Microorganisms

Gluconobacter $\mathrm{sp}$. W-315 was isolated and identified as described in the following paper ${ }^{1)}$. Neurospora crassa IFO 6068 and other microorganisms used were from the collection of this laboratory.

Bacterial Growth FPW-4.

Bacterial growth was measured by absorbancy at $660 \mathrm{~nm}$ using a Hitachi photoelectric colorimeter

Determination of Antibiotic Activity

Antibiotic activity was measured by agar dilution method ${ }^{3)}$ or pulp disc method ${ }^{3)}$ at $30^{\circ} \mathrm{C}$ for 24 hours. The amount of the compound in fermentation broth was also determined spectrophotometrically by absorbance at $370 \mathrm{~nm}$ using a Hitachi 124 spectrophotometer.

Thin-layer Chromatography of the Antibiotic

Crude powder of the antibiotic was purified with preparative thin-layer chromatography (TLC) on Silica gel 60 plate (Merck) using a solvent system of chloroform - methanol - acetic acid, 65: 5: 1. AB-315 obtained from preparative TLC was further purified by reversed-phase TLC with Silica gel 60 silanised plate (Merck) using a solvent system of acetonitrile - acetic acid - water, 40: 0.4: 60 .

High-performance Liquid Chromatography

High-performance liquid chromatography (HPLC) of the antibiotic was performed using a Toyo 
Soda HLC-803 with a TSK-GEL LS-410K column and the detection was carried out using a UV detector Uvilog-5 II (Ōyō-Bunkō Kiki Co., Ltd).

Assay of Antitumor Activity

Assay of antitumor activity of the antibiotic was kindly carried out by Dr. T. Tsuruo. KB cells derived from a human epidermoid carcinoma were grown on an EAGLE's minimal essential medium supplemented with $10 \%$ of calf serum. Antibiotic concentration required to decrease $50 \%$ of total cell number was compared to that of the control and was expressed as $\mathrm{ED}_{50}$. $\mathrm{ED}_{50}$ less than $20 \mu \mathrm{g} / \mathrm{ml}$ was regarded as an active antitumor compound.

Other Experimental

UV Spectrum of the antibiotics and IR spectrum of the fraction 2 was measured using a Hitachi 124 spectrophotometer and a Jasco IRA-1 spectrometer, respectively. Melting points were measured on a Yanagimoto apparatus MP-S3 and not corrected. Optical rotation in methanol was measured on a Jasco digital polarimeter DIP-4. Crystallization of the antibiotic was done from the concentrated solution in ethyl acetate.

\section{Results}

\section{Production of the Antibiotic}

The production of the antibiotic consists of two-step fermentations. The first step is the production of spent medium of Neurospora crassa and the $2 \mathrm{nd}$ is the production of the antibiotic in the spent medium.

\section{1st Step}

Neurospora crassa was inoculated from the slant culture of MY agar medium ( $30 \mathrm{~g}$ malt extract, $5 \mathrm{~g}$ yeast extract and $15 \mathrm{~g}$ of agar in 1 liter of distilled water, $\mathrm{pH}$ 5.6) into a 500-ml culture flask containing $100 \mathrm{ml}$ of Czapek-Dox medium (20 g sucrose, $3 \mathrm{~g} \mathrm{NaNO}_{3}, 1 \mathrm{~g} \mathrm{~K}_{2} \mathrm{HPO}_{4}, 0.5 \mathrm{~g} \mathrm{KCl}, 0.5 \mathrm{~g}$ $\mathrm{MgSO}_{4} \cdot 7 \mathrm{H}_{2} \mathrm{O}, 0.018 \mathrm{~g} \mathrm{FeSO} \cdot \cdot 7 \mathrm{H}_{2} \mathrm{O}$ in 1 liter of distilled water, $\mathrm{pH} \mathrm{6.5).} \mathrm{After} \mathrm{growth} \mathrm{at} 30^{\circ} \mathrm{C}$ for 48 hours on a reciprocal shaker, $10 \mathrm{ml}$ of the preculture was inoculated into a 3-liter culture flask containing 1 liter of the same medium. After growth at $30^{\circ} \mathrm{C}$ for 5 to 7 days on the reciprocal shaker, the broth was filtered through a Toyo filter paper No. 525. This filtration was not necessary for the preparation of the antibiotic production, but when filtration was done, isolation of the antibiotic was easier than that without filtration.

\section{2nd Step}

Overnight culture ( $5 \mathrm{ml}$ in a test tube) in nutrient broth of Gluconobacter $\mathrm{sp}$. W-315 was inoculated into a 3-liter culture flask containing $400 \mathrm{ml}$ of the spent medium of Neurospora crassa obtained as above which had been sterilized at $110^{\circ} \mathrm{C}, 10$ minutes. The $\mathrm{pH}$ of the medium was adjusted to 7.2 before sterilization and the culture was carried out at $30^{\circ} \mathrm{C}$ on the reciprocal shaker. The time courses of the antibiotic production and bacterial growth were shown in Fig. 1. Almost no antibiotic was detected before incubation of 12 hours but it increased rapidly with a linear rate until 36 hours, giving the maximum activity at approximately 48 hours. The amount of antibiotic then decreased gradually. It is known that antibiotic production in bacilli, fungi and actinomycetes is stimulated during sporulating phase ${ }^{2}$. The antibiotic production of Gluconobacter sp. W-315, however, occurred during its vegetative growth and not in the late stationary phase, although this strain is not a spore former. The $\mathrm{pH}$ increased from 7.2 to 7.8 during incubation time. When the initial $\mathrm{pH}$ was lowered to 6.5 , essentially no antibiotic production occurred and cell growth also decreased to approximately one tenth. 
Fig. 1. Antibiotic production as a function of growth.

The amount of antibiotic was measured by absorbance at $370 \mathrm{~nm}$ of the culture medium because the antibiotic showed $\mathrm{UV}_{\max }$ at $370 \mathrm{~nm}$ and no other material showing absorbance at this wavelength was detected in the fermentation broth.

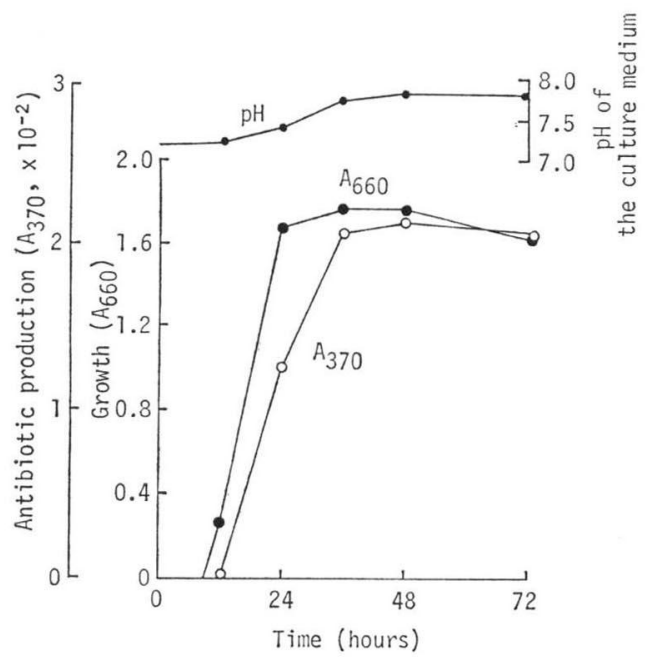

Isolation and Purification of the Antibiotic

The culture supernatant obtained by centrifugation was extracted with diethyl ether after acidification with $\mathrm{HCl}$ to $\mathrm{pH}$ 2. Antibiotic dissolved in diethyl ether was then extracted by $1 \% \mathrm{NaHCO}_{3}$. Aqueous solution was acidified to $\mathrm{pH} 2$ yielding the precipitates of the antibiotic. The crude powder of antibiotic was obtained by drying the precipitate in vacuo. One liter of culture yielded $\mathrm{ca} .100 \mathrm{mg}$ of crude powder with yellow-green fluorescence. Preparative TLC was performed by applying the crude antibiotic dissolved in acetone onto the plate and developing with the solvent system of chloroform-methanol - acetic acid, 65: 5: 1 .

The compound was developed as nearly one main yellow spot on the plate and almost all the antimicrobial activity was detected on this spot. On prolonged fermentation, however, two major spots and a few minor spots were

observed suggesting that the degradation of the antibiotic had occurred in the fermentation process. Main spot having more than $95 \%$ of antimicrobial activity was scraped off from TLC plate, extracted with methanol and dried in vacuo. This fraction obtained by preparative TLC as one spot was named as AB-315. AB-315 was then applied to analytical HPLC. Elution profile is shown in Fig. 2. At least, 8 fractions were detected. Since the relative height of absorbance of each fraction fluctuated in every trial, a possibility existed that many fractions separated by HPLC were artifacts caused by acidification of the supernatant before ether extraction. To examine this possibility, we applied the cell-free supernatant of the culture broth to HPLC with and without pre-treatment of

Fig. 2. HPLC chromatogram of antibiotic AB-315.

AB-315 $(10 \mu \mathrm{g})$ dissolved in methanol $(10 \mu \mathrm{l})$ was loaded on the column $(4 \mathrm{mmID} \times 30 \mathrm{~cm})$ and eluted at $0.5 \mathrm{ml}$ per minute using a solvent system of methanol - formic acid - water, 200: 0.84: 80 .

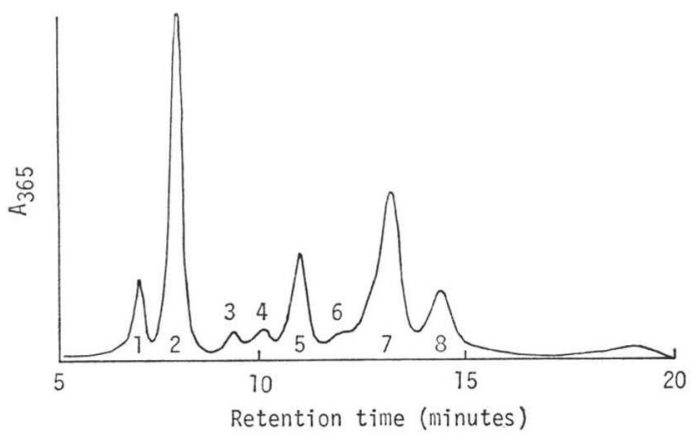

hydrochloric acid. Antibiotics produced in the

Fig. 3. Reversed-phase TLC of AB-315 with silanised Silica gel 60.

AB-315 was developed with the solvent system of acetonitrile-acetic acid-water, 40:0.4:60. Numbers in Figure correspond to the peak number in HPLC shown in Fig. 2.

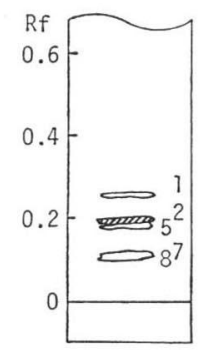


fermentation broth consisted of one main compound accompanied with other minor compounds. After treatment with hydrochloric acid, parts of the major compound (fraction 2) was converted to other fractions. From these results, we concluded that the fraction 2 was the main component of the antibiotics.

Reversed-phase TLC with Silica gel 60 silanised plate was performed as the last step of purification as shown in Fig. 3. These bands were scraped off separately from the plate, extracted with methanol and dried in vacuo and were then identified by HPLC. One liter of culture yielded $c a .5 \mathrm{mg}$ of fraction 2 with yellow fluorescence. The yields of other fractions were less than that of fraction 2.

Determination of Relative Molecular Sizes of Fractions in AB-315

As shown in Fig. 2, antibiotic AB-315 can be separated at least to 8 fractions by HPLC. To obtain the relative molecular sizes of these fractions in AB-315, gel filtration was carried out with Sephadex LH-20 by recycling the eluate four times with methanol as solvent (Fig. 4A). The elution profile was nearly symmetric but broad. We performed HPLC with fractions indicated with arrows

Fig. 4. Determination of relative molecular sizes of fractions in AB-315.

(A) Gel filtration profile of AB-315 on Sephadex LH-20 recycled four times with methanol as a solvent. Gel filtration was carried out with a column $(1.9 \times 31 \mathrm{~cm})$ and each $0.7 \mathrm{ml}$ fraction was collected.

(B) Distribution of major 4 fractions in the gel eluate as revealed by HPLC. Fractions indicated by arrows from a to $\mathrm{j}$ shown in Fig. 4A were analyzed by HPLC. From the HPLC chromatograms, the amounts of the major 4 fractions (fractions 1, 2, 5 and 7) in each sample were analyzed and plotted against elution volume of each sample.
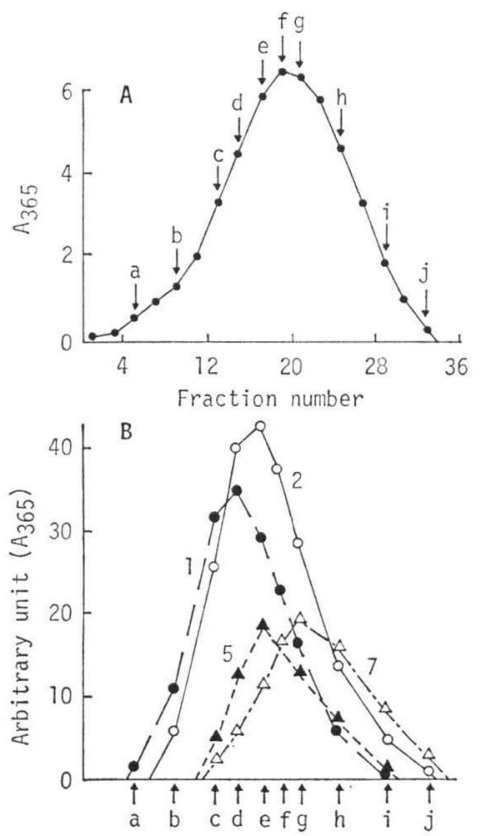

in Fig. 4A and obtained distribution of the major 4 fractions in the gel eluate. It is shown in Fig. 4B. Fraction 1 was eluted first on gel filtration and fraction 7 was the last in these four fractions. From these results, we assigned the order of relative molecular size of each fraction as follows; $1>2 \fallingdotseq 5>7$.

Physicochemical Properties of the Antibiotics

UV Spectra of AB-315 and five major fractions dissolved in methanol are shown in Fig. 5. They have $U V_{\max }$ at $365 \mathrm{~nm}$ and shoulder at $383 \mathrm{~nm}$ and a small peak at $268 \mathrm{~nm}$. The yellow color and

Fig. 5. UV spectra of antibiotics in methanol. Concentrations of the antibiotics were adjusted to $9.5 \mu \mathrm{g} / \mathrm{ml}$.

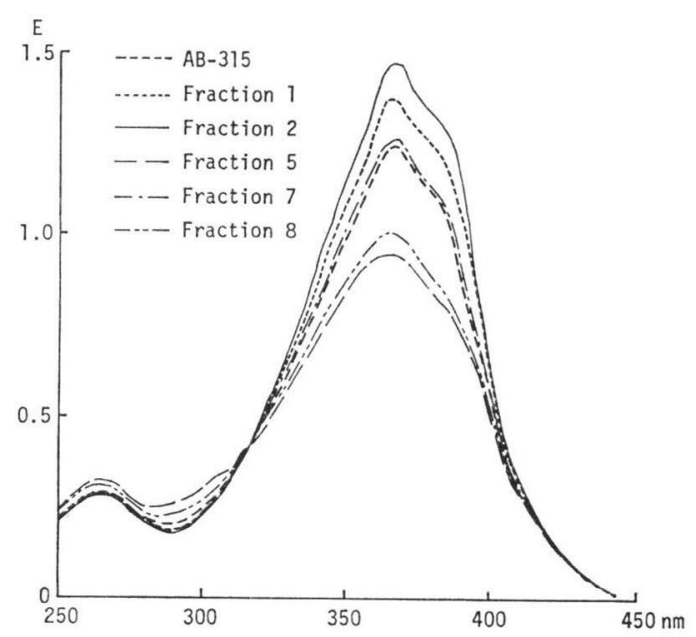


Fig. 6. IR Spectrum of fraction $2(\mathrm{KBr})$.

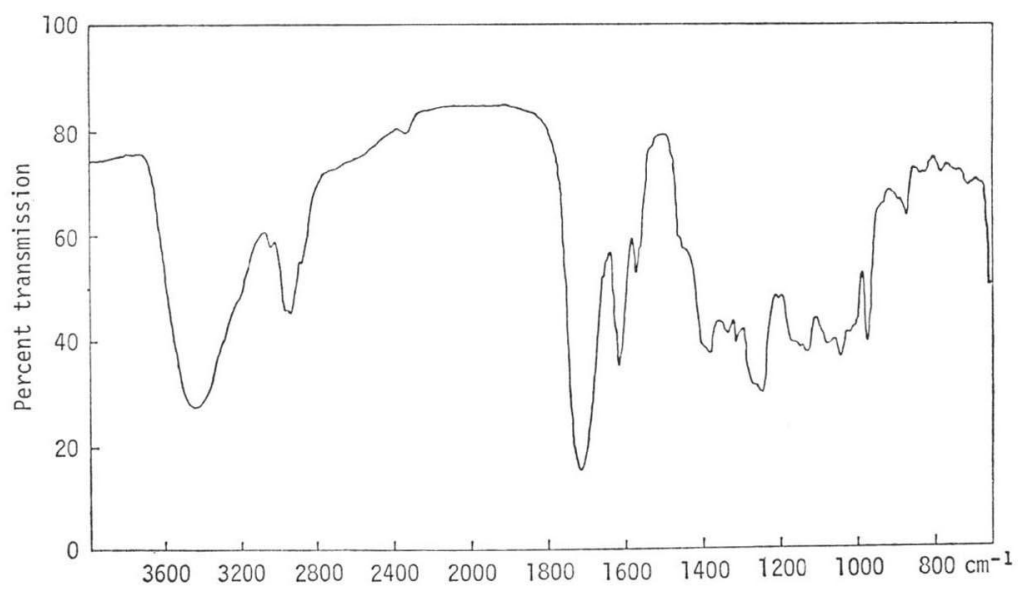

the broad absorption maxima at $365 \mathrm{~nm}$ clearly indicate a polyenic nature of these compounds. The IR spectrum of fraction 2 in $\mathrm{KBr}$ tablet is shown in Fig. 6. AB-315 is readily soluble in acetone or ethyl acetate but purified fraction 2 became nearly insoluble in these solvents. Fraction 2 reduced potassium permanganate and became purple upon treatment with sulfuric acid but no positive reactions can be seen with ninhydrin, ferric chloride or silver nitrate suggesting that amino or imino group, hydroxybenzene and sugar moiety were absent. Fraction 2 melted at $149 \sim 150^{\circ} \mathrm{C}$, gave $[\alpha]_{\mathrm{D}}^{20}+29.1^{\circ}$ and sulfur, phosphorus or halogen could not be detected as constituent. Since the antibiotic fraction 2 contains a small amount of dichlorodimethylsilane derived from silanised Silica gel plate, we could not obtain the exact values of elemental analysis.

\section{Biological Properties of the Antibiotics}

Acute toxicity of AB-315 was kindly assayed by Dr. M. NAKAmura. LD L $_{50}$ (in mice, i.p.) was $c a .150 \mathrm{mg} / \mathrm{kg}$. This value was less toxic compared to those of polyenic compounds previously reported $^{4)}$. Antitumor activities of the antibiotics (crude powder, AB-315 and fraction 2) assayed by growth inhibition of KB cells derived from human epidermoid carcinoma are all under the criteria of effectiveness. The antimicrobial spectra have been determined by the agar dilution method (Table 1). It has been shown that the antibiotic was active against Gram-positive and -negative bacteria, slightly active against fungi but inactive against yeasts. Both AB-315 and fraction 2 had the potent activity against all bacteria tested. However, the other fractions such as fractions 1, 5 and 7 were less active than fraction 2 although they had considerable antibacterial activity.

When fraction 2 was added to the growing cells of $E$. coli $\mathrm{K}-12$ in nutrient broth, viable cells decreased exponentially. This decrease in viability was dependent on the concentration of the antibiotic. The bactericidal action of fraction 2 was not observed under non-growing conditions such as in phosphate buffer.

\section{Discussion}

It is well known that polyenic antibiotics could inhibit the growth of fungi and yeasts. Some of them, however, could not only inhibit the growth of fungi and yeasts, but also some bacteria. Re- 
Table 1. Minimum inhibitory concentration ${ }^{a)}$ of various antibiotics (agar dilution method).

\begin{tabular}{|c|c|c|c|c|c|}
\hline \multirow{2}{*}{ Test organism } & \multirow{2}{*}{ AB-315 } & \multicolumn{4}{|c|}{ Fraction } \\
\hline & & 1 & 2 & 5 & 7 \\
\hline Escherichia coli $\mathrm{K}-12$ & 7.5 & 10 & 5 & $>10$ & 7 \\
\hline Escherichia coli B IID & 0.5 & N.T. & N.T. & N.T. & N.T. \\
\hline Salmonella typhimurium 7M1 & 10 & 50 & 10 & $>10$ & 10 \\
\hline Pseudomonas fluorescens IFO 3081 & 10 & 20 & 7 & $>10$ & 10 \\
\hline Pseudomonas aeruginosa IAM 1007 & 50 & N.T. & N.T. & N.T. & N.T. \\
\hline Erwinia aroideae IAM 1024 & 5 & 7 & 1 & 20 & 10 \\
\hline Serratia marcescens FK 5 & 50 & N.T. & N.T. & N.T. & N.T. \\
\hline Klebsiella aerogenes ATCC 7256 & 5 & 10 & 1 & $>10$ & 10 \\
\hline Proteus mirabilis AHU 1468 & 7.5 & 15 & 5 & 10 & 10 \\
\hline Bacillus megaterium & 10 & 5 & 1 & $>10$ & 5 \\
\hline Bacillus subtilis Marburg & 50 & 20 & 10 & $>10$ & 10 \\
\hline Staphylococcus aureus $209 \mathrm{P}^{\mathrm{e})}$ & 50 & 20 & 20 & N.T. & N.T. \\
\hline Micrococcus luteus IAM 1097 & 10 & 10 & 10 & $>10$ & 10 \\
\hline Micrococcus luteus IFO 3232 & 20 & 20 & 10 & N.T. & N.T. \\
\hline Streptococcus faecalis ATCC 8043 & 1 & N.T. & N.T. & N.T. & N.T. \\
\hline Streptomyces sp. ${ }^{\mathrm{b})}$ & 0.8 & N.T. & N.T. & N.T. & N.T. \\
\hline Neurospora crassa IFO $6068^{\mathrm{c})}$ & $>200$ & $>200$ & $>200$ & N.T. & N.T. \\
\hline Saccharomyces cerevisiae $e^{\mathrm{d})}$ & $>500$ & $>500$ & $>500$ & N.T. & N.T. \\
\hline
\end{tabular}

a) $\mathrm{MIC}$ in $\mu \mathrm{g} / \mathrm{ml}$. Heart Infusion Agar was used except that ${ }^{\mathrm{b})}$; Bennet medium, ${ }^{\mathrm{c})}$; Czapek-Dox medium, and ${ }^{\text {d) }}$; Henneberg medium + yeast extract.

e) Incubated at $37^{\circ} \mathrm{C}$ for 24 hours.

N.T.: not tested

sistaphylin has been reported to inhibit Gram-positive bacteria ${ }^{4}$. Flavomycoin and flavofungin are carbonyl-conjugated pentaene compounds produced by genus Streptomycetes, but they are inactive against bacteria ${ }^{5,8)}$.

Recently, it has been reported that polyenic antibiotics produced by genus Bacilli, namely aurantinin and proticin, were respectively active against Gram-positive and Gram-negative bacteria ${ }^{7 \sim 9)}$. The antibiotic described in this paper is clearly different from these antibiotics, because aurantinin and proticin were shown to be triene and proticin contained phosphorus in its structure. Since the polyenic antibiotic active against both Gram-positive and Gram-negative bacteria has not been reported thus far, $\mathrm{AB}-315$ and fractions 1, 2, 5 and 7 are the first antibiotics showing a polyenic nature isolated from a culture of the genus Gluconobacter. They are active against Gram-positive and Gram-negative bacteria, slightly active against fungi but not against yeasts.

Antibiotic production by genus Gluconobacter is unusual and the mode of its production is very interesting. Two-step fermentations applied in this antibiotic production were essential at present. Examination of the nutritional elements required for antibiotic production in the spent medium of Neurospora crassa will be discussed in the following paper. ${ }^{1)}$ Antibiotic AB-315 can be separated at least to 8 fractions by HPLC and the majority of them, fractions 1, 2, 5, 7 and 8 were isolated by silanised TLC. UV Profiles of AB-315 and these 5 fractions resembled each other (Fig. 5). Profiles on gel filtration of AB-315 recycled four times indicate that molecular weight of 4 fractions differs from each other except that fraction 2 and fraction 5 which have similar molecular weights. However, their differences in molecular weights are considered to be very small because they could not be separated even when recycled four times on Sephadex LH-20. These results clearly indicate that antibiotic AB-315 was a mixture of chemically related compounds with a polyenic nature. 


\section{Acknowledgements}

We are grateful to Dr. Hiroo AokI, Faculty of Agriculture, Meijo University, for his helpful discussion on chemical properties of the antibiotic. We wish to thank Dr. Masataka NAKAmura, Tohoku University School of Medicine, for estimating acute toxicity, and Dr. TAKASHI Tsuruo, Cancer Chemotherapy Centre, for assaying antitumor activity.

\section{References}

1) Watanabe, T.; K. Izaki \& H. TAKahashi: New polyenic antibiotics active against Gram-positive and -negative bacteria. II. Screening of antibiotic producers and taxonomical properties of Gluconobacter sp. W-315. J. Antibiotics 35: $1148 \sim 1155,1982$

2) Balassa, G.: The gentic control of spore formation in Bacilli. Curr. Top. Microbiol. Immunol. 56: $99 \sim 192,1971$

3) Tanaka, N. \& S. Nakamura: Kōseibusshitsu Taiyo. 2nd ed., p. 23 29, Tokyo Univ. Press, Tokyo, 1967 (in Japanese)

4) Hamilton-Miller, J. M. T.: Chemistry and biology of the polyene macrolide antibiotics. Bacteriol. Rev. 37: 166 196, 1973

5) Schlegel, R. \& H. Thrum: A new polyene antibiotic, flavomycoin structural investigations. II. J. Antibiotics 24: 368 374, 1971

6) Bognar, R.; B. O. Brown, W. J. S. Lockley, S. Makleit, T. P. Toube, B. C. L. Weedon \& K. Zsupan: The structure of flavofungin. Tetrahedron Lett. 1970: 471 474, 1970

7) Nishikiori, T.; R. Masuma, R. ÖIwa, M. Katagiri, J. Awaya, Y. Iwai \& S. Ōmura: Aurantinin, a new antibiotic of bacterial origin. J. Antibiotics 31: 525 532, 1978

8) PräVE, P.; D. SUKATSCH \& L. VéRtesY: Proticin, a new phosphorus-containing antibiotic. I. Taxonomy, fermentation, isolation, and biological properties. J. Antibiotics 25: 1 3, 1972

9) VÉRTESY, L.: Proticin, a new phosphorus-containing antibiotic. II. Characterization and chemical studies. J. Antibiotics 25: 4 10, 1972 